\title{
Temperature and magnetic-field dependence of the elastic constants of Ni-Mn-Al magnetic Heusler alloys
}

\author{
Xavier Moya, Lluís Mañosa,* and Antoni Planes \\ Departament d'Estructura i Constituents de la Matèria, Facultat de Física, Universitat de Barcelona, Diagonal 647 , \\ E-08028 Barcelona, Catalonia, Spain
}

Thorsten Krenke and Mehmet Acet

Fachbereich Physik, Experimentalphysik, Universität Duisburg-Essen, D-47048 Duisburg, Germany

Michel Morin

Groupe d'Etudes de Métallurgie Physique et Physique des Matériaux, INSA, 20, Av. A. Einstein, 69621 Villeurbanne, France

J. L. Zarestky and T. A. Lograsso

Ames Laboratory, Department of Physics, Iowa State University, Ames, Iowa 50011, USA

(Received 20 April 2006; published 21 July 2006)

\begin{abstract}
We report on measurements of the adiabatic second-order elastic constants of the off-stoichiometric $\mathrm{Ni}_{54} \mathrm{Mn}_{23} \mathrm{Al}_{23}$ single-crystalline Heusler alloy. The variation in the temperature dependence of the elastic constants has been investigated across the magnetic transition and over a broad temperature range. Anomalies in the temperature behavior of the elastic constants have been found in the vicinity of the magnetic phase transition. Measurements under applied magnetic field, both isothermal and variable temperature, show that the value of the elastic constants depends on magnetic order, thus giving evidence for magnetoelastic coupling in this alloy system.
\end{abstract}

DOI: 10.1103/PhysRevB.74.024109

PACS number(s): 62.20.Dc, 81.30.Kf, 64.70.Kb

\section{INTRODUCTION}

Among bcc-based solids undergoing martensitic transformations, magnetic shape memory alloys have received much attention in recent years due to their distinct properties arising from the coupling between structure and magnetism. ${ }^{1}$ This coupling, which results in magnetic-field-induced strains, makes these materials candidates for potential use in device applications. This effect occurs in ferromagnetic systems undergoing a martensitic transformation and leads to the magnetic shape memory (MSM) effect. It originates from the reorientation of martensite domains and/or from the magnetic-field-induced austenite-martensite transformation. ${ }^{2}$ Intimately related to magnetic shape memory is the magnetocaloric effect (MCE). ${ }^{3,4}$ The fact that in some alloys the magnetization of the martensitic phase is lower than that in the parent phase leads to an inverse magnetocaloric effect. ${ }^{5}$

The prototype MSM system Ni-Mn-Ga has been most extensively studied because of the presence of magnetic-fieldinduced strains as large as $10 \% \cdot{ }^{6,7}$ From a fundamental point of view, this alloy exhibits singular lattice-dynamical behavior. Specifically, it has been shown that the transverse $\mathrm{TA}_{2}$ branch shows a dip (anomalous phonon) at a particular wave number, at which the energy softens with decreasing the temperature. ${ }^{8-10}$ The temperature dependence of the energy of the anomalous phonon parallels that of the shear elastic constants, which also soften with decreasing temperature. ${ }^{11-13}$ Significant magnetoelastic coupling exists in this system as evidenced by the enhancement of the anomalous phonon softening when the sample orders ferromagnetically, $9,10,14$ and by the change in the elastic constants when a magnetic field is applied at constant temperature. ${ }^{15}$
The brittleness of the Ni-Mn-Ga compound has prompted the search for MSM materials with mechanically more favorable properties such as $\mathrm{Ni}-\mathrm{Mn}-\mathrm{Al}$. Stoichiometric $\mathrm{Ni}_{2} \mathrm{MnAl}$ is structurally stable down to low temperatures, but martensitic transformations occur within a certain range of compositions close to stoichiometry. ${ }^{16}$ The low-temperature martensitic structure depends on composition, and the observed structures are the same as those reported for other Ni-Mnbased magnetic shape memory alloys. ${ }^{16-20}$ Previous studies have revealed that at low temperatures, $\mathrm{Ni}_{2} \mathrm{MnAl}$ consists of a mixed $L 2_{1}+B 2$ phase, which incorporates ferromagnetic and antiferromagnetic parts. ${ }^{21-23}$ The magnetic ordering in the metastable $B 2$ phase of $\mathrm{Ni}_{2} \mathrm{MnAl}$ is conical antiferromagnetic, ${ }^{24}$ and the presence of the $B 2$ phase in this mixed state is due to the low $B 2-L 2_{1}$ order-disorder transition as compared to that of Ni-Mn-Ga. This leads to slow kinetics for the ordering process. ${ }^{22}$

Recently, we have carried out elastic and inelastic neutron scattering experiments in a Ni-Mn-Al single crystal, for which we observe significant $\mathrm{TA}_{2}$ phonon softening at $\xi_{0}$ $=0.33 .{ }^{25}$ In addition, a number of elastic satellites associated with magnetic ordering and with structural instabilities were also observed. It has been shown that these precursor satellites only grow once the sample orders magnetically, suggesting that there is an interplay of magnetism and structure in Ni-Mn-Al at a microscopic level.

The aim of the present work is to extend the study of the lattice dynamics of the $\mathrm{Ni}-\mathrm{Mn}-\mathrm{Al}$ system to the longwavelength limit by measuring the elastic constants. We pay special attention to the effect of applied magnetic field and provide information on the interplay between structure and magnetism in the long-wavelength limit. 


\section{EXPERIMENTAL DETAILS}

The sample investigated is a single crystal with composition $\mathrm{Ni}_{54} \mathrm{Mn}_{23} \mathrm{Al}_{23}$ grown by the Bridgman method; the estimated error in the composition is \pm 1 at. \%. Details of sample preparation are given in Ref. 25. The single crystal used in the present study was cut from the larger single crystal used in previous neutron diffraction experiments at the High Flux Isotope Reactor (HFIR) at the Oak Ridge National Laboratory (ORNL). ${ }^{25}$ It was oriented using the $\mathrm{x}$-ray Laue backscattering technique. From the original rod, a parallelepiped specimen with dimensions $8.70 \times 10.25 \times 10.75 \mathrm{~mm}^{3}$ with faces parallel to the $(1 \overline{1} 0)$, (110), and (001) planes, respectively, was cut using a low-speed diamond saw. A thinner sample $(2.45 \mathrm{~mm}$ thick) with faces parallel to the (1 $\overline{1} 0)$ plane was also cut from the original rod in order to measure slow modes, which may be affected by strong attenuation. In addition, small oriented pieces cut from the ingot close to the extracted parallelepiped specimen were cut for magnetization measurements.

The velocity of ultrasonic waves was determined by the pulse-echo technique. X-cut and Y-cut transducers with resonant frequencies of $10 \mathrm{MHz}$ were used to generate and detect the ultrasonic waves. The transducers were acoustically coupled to the surface of the sample by means of Nonaq stopcock grease in the temperature range $10-250 \mathrm{~K}$, by Dow Corning Resin 276-V9 in the temperature range 210-320 K, and by Crystalbond509 (Aremco Products, Inc.) in the temperature range $310-390 \mathrm{~K}$.

A standard closed-cycle helium refrigerator (displex) was used for measurements below room temperature and up to $300 \mathrm{~K}$. The temperature inside the displex was measured with a silicon diode. For high-temperature measurements (up to $390 \mathrm{~K}$ ) the sample was placed into a copper sample holder which was heated by means of a heating plate. In this case, the temperature was measured with a Pt-100 resistor embedded into the sample holder in close proximity to the sample.

A device has been constructed that allows both isofield and isothermal measurements of the ultrasonic velocities. The sample and transducer are placed into a copper sample holder equipped with a Peltier element. The sample is placed on the upper surface of the Peltier element, of which the bottom surface is in good thermal contact with the copper sample holder. The sample holder is placed between the poles of an electromagnet and lies on top of a copper cylinder. The copper cylinder is partially immersed into a bath of ice and water. The temperature is measured by a thermocouple attached to the sample. By controlling the current input into the Peltier element it is possible to achieve a finetuning of temperature within the range $250-310 \mathrm{~K}$. Temperature oscillations are less than $0.1 \mathrm{~K}$. The gap between the magnetic poles is $28 \mathrm{~mm}$ which enables fields up to $13 \mathrm{kOe}$ to be applied perpendicular to the propagation direction of the ultrasonic waves.

The magnetization was measured as a function of temperature $(4-300 \mathrm{~K}$ ) and field (up to $5 \mathrm{~T}$ ) using a superconducting quantum interference device magnetometer.
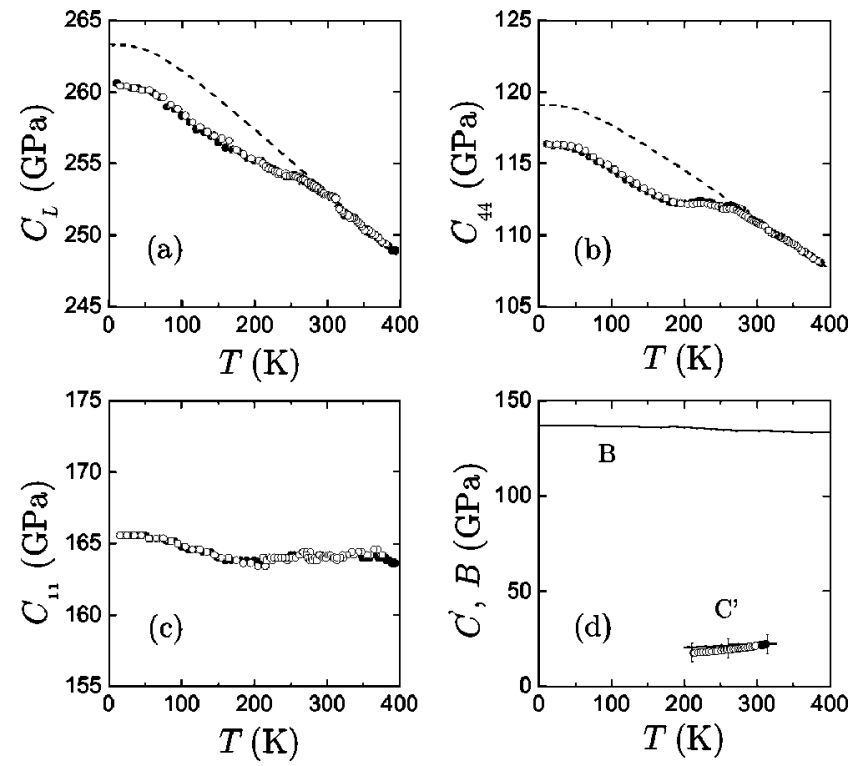

FIG. 1. Temperature dependence of the elastic moduli (a) $C_{L}$, (b) $C_{44}$, and (c) $C_{11}$. Cooling and heating runs are represented by open and solid symbols, respectively. The dashed lines show the expected behavior for a Debye solid. (d) shows the values of the bulk modulus $(B)$ and the shear elastic constant $\left(C^{\prime}\right)$ calculated from the complete set of elastic constants measured (solid lines). The values of $C^{\prime}$ measured from the thinner sample are also shown for comparison.

\section{EXPERIMENTAL RESULTS AND DISCUSSION}

\section{A. Temperature dependence of the elastic constants}

For the parallelepiped sample, we have measured the velocity of the longitudinal and shear waves propagating along the [001] direction. Along the [110] direction, we have also measured longitudinal and [001]-polarized waves. Due to strong attenuation, it has not been possible to obtain reliable echoes for the $[1 \overline{1} 0]$-polarized shear waves. For such a mode, it was possible to obtain echoes on the thinner sample. The values of the elastic constants at room temperature derived from these measurements are $C_{L}=253 \pm 3 \mathrm{GPa}, C_{44}$ $=111 \pm 2 \mathrm{GPa}, C_{11}=164 \pm 3 \mathrm{GPa}$, and $C^{\prime}=21 \pm 4 \mathrm{GPa}$. It is worth noting that the value of $C^{\prime}$ computed using the data obtained on the large crystal, $C^{\prime}=C_{44}+C_{11}-C_{L}=22 \pm 8 \mathrm{GPa}$, is in good agreement with the value obtained for the smaller sample. Moreover, present values are in good agreement with those obtained from the initial slopes of the acoustic phonon branches recently measured by inelastic phonon scattering. ${ }^{25}$

From the temperature dependence of ultrasonic waves along the [110] and [001] directions, we have computed the temperature dependence of three independent elastic constants $C_{L}, C_{44}$, and $C_{11}$. These are shown in Figs. 1(a)-1(c), respectively. The data correspond to cooling (open circles) and heating (solid circles) runs and are obtained as an average over several independent runs. From previous neutron scattering measurements, the change of the cubic unit cell parameter between $10 \mathrm{~K}$ and $350 \mathrm{~K}$ was estimated to be about $0.3 \%$. Therefore, no corrections for temperature- 
induced changes of the samples dimensions were taken into account. All elastic constants increase with decreasing temperature and saturate at low temperature. It should be noted that $C_{11}$ is almost independent of temperature. This behavior is similar to that reported for Ni-Mn-Ga. ${ }^{13}$ Dashed lines in Figs. 1(a) and 1(b) represent the expected temperature behavior described by the quasiharmonic approximation of lattice dynamics using the Debye model. ${ }^{26}$ Within this framework, the temperature dependence of the elastic constants is given by

$$
C_{i j}=C_{i j}^{0}\left[1-\kappa F\left(T / \theta_{D}\right)\right]
$$

where

$$
F\left(T / \theta_{D}\right)=3\left(T / \theta_{D}\right)^{4} \int_{0}^{T / \theta_{D}} \frac{x^{3}}{e^{x}-1} d x
$$

$C_{i j}^{0}$ is the elastic constant at zero temperature, $\theta_{D}$ the Debye temperature, and $\kappa$ a material-dependent parameter. It is seen that the measured elastic constants show an anomalous temperature dependence deviating from the Debye behavior within the temperature range 200-300 K, approximately.

Figure 1(d) shows the temperature dependence of the bulk modulus $(B)$ and the shear elastic constant $\left(C^{\prime}\right)$ calculated from the complete set of measured elastic constants (solid lines). The values of $C^{\prime}$ obtained from the ultrasonic velocity measured on the thinner sample are also shown for comparison. We note that the strong attenuation of the shear waves associated with this mode makes it difficult to perform an accurate measurement of this elastic constant, and the values are affected by considerable error as shown by the error bars in Fig. 1(d). It was also not possible to obtain reliable echoes below $200 \mathrm{~K}$. The measured and calculated values are in good agreement within experimental error. It should be noted that $C^{\prime}$ exhibits a low value and softens with decreasing temperature. Such a temperature softening is typical for bccbased solids which undergo martensitic transformations ${ }^{27}$ and reflects the dynamical instability of the cubic lattice against shearing of (110) planes along the $(1 \overline{1} 0)$ direction. It is worth noting that the elastic anisotropy $A=C_{44} / C^{\prime}=5.3$ is significantly lower than for $\mathrm{Cu}$-based prototypical shape memory alloys. ${ }^{28}$

Previous studies of polycrystalline Ni-Mn-Al samples with composition close to the studied sample revealed a magnetic transition occurring around $300 \mathrm{~K} .{ }^{22}$ This temperature corresponds to the start of the deviation of the measured elastic constants from Debye behavior. This suggests that the observed deviation from Debye behavior may be due to the development of magnetic order. In addition, neutron scattering experiments also revealed the development of magnetic ordering below $\sim 300 \mathrm{~K}$ for the crystal used in the present experiments.

Recent ab initio calculations for stoichiometric $\mathrm{Ni}_{2} \mathrm{MnAl}$ have reported a value of the bulk modulus $B=157 \mathrm{GPa}$ and $B=141 \mathrm{GPa}$ for ferromagnetic and antiferromagnetic ordering, respectively. ${ }^{29}$ The value derived from the present ultrasonic measurements at $T=10 \mathrm{~K}$ is in good agreement with the computed value for antiferromagnetic order.
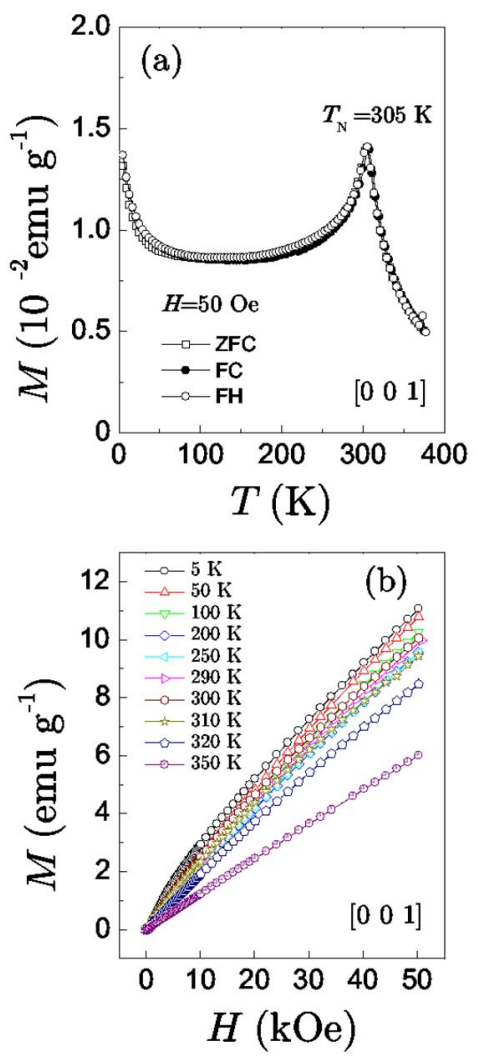

FIG. 2. (Color online) (a) ZFC, FC, and FH $M(T)$ in a field of $H=50$ Oe applied along the [001] crystallographic direction. (b) Magnetization versus field at several temperatures above and below the magnetic transition. Prior to each $M(H)$ measurement, the samples were prepared in the ZFC state by bringing them above $380 \mathrm{~K}$. Solid lines are guides to the eye.

\section{B. Magnetization measurements}

With the aim of characterizing the magnetic behavior of the alloy, we have performed magnetization measurements as a function of the temperature and magnetic field. Figure 2(a) shows the temperature dependence of the magnetization $M(T)$ in a low external magnetic field $(H=50 \mathrm{Oe})$ applied along the [001] crystallographic direction in the temperature range $5 \mathrm{~K} \leqslant T \leqslant 380 \mathrm{~K}$. Prior to the measurements, the sample was prepared in a zero-field-cooled (ZFC) state by cooling it from $380 \mathrm{~K}$ to $5 \mathrm{~K}$ in the absence of a magnetic field. Subsequently, the external field was applied and the measurements were taken on increasing temperature up to $380 \mathrm{~K}$. Then, without removing the external field, the measurement was made on decreasing temperature-i.e., fieldcooled (FC). As a last step, again without removing the external field, the magnetization was measured on increasing temperature. The last step is denoted as the field-heated (FH) sequence. FC and FH curves retrace each other, showing that no structural transition occurs in the studied sample. The magnetization exhibits a peak at $305 \mathrm{~K}$ which corresponds to the magnetic transition from the paramagnetic state to the mixed ferromagnetic-antiferromagnetic state. ${ }^{21}$ This temperature agrees well with the temperature below which magnetic satellites have been observed by neutron diffraction. 

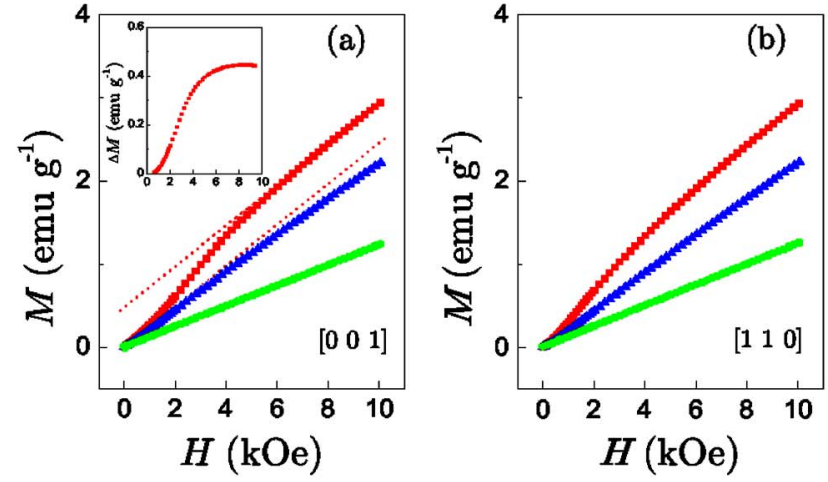

Due to the absence of any considerable splitting between ZFC and FC curves together with the low value of the magnetization, it is expected that the ordering in the sample is mainly antiferromagnetic. This behavior is in agreement with previous studies on polycrystalline samples with composition close to the studied sample. ${ }^{22}$ The upturn of the magnetization below $130 \mathrm{~K}$ could be related to a reorientation of the conical structure, as was stated in Ref. 21

To further characterize the magnetic properties, the magnetic-field dependence of the magnetization $M(H)$ was measured in fields up to $50 \mathrm{kOe}$ applied along the [001], [110], and [1 $\overline{1} 0]$ directions. In Fig. 2(b), we illustrate the behavior obtained at selected temperatures for the field in the [001] direction. The magnetization versus field data at $350 \mathrm{~K}$, which correspond to the paramagnetic state, show typical linear behavior of a paramagnet. Similar behavior is observed for the $M(H)$ data at $320 \mathrm{~K}$. However, in this case the data deviate slightly from linearity because of the presence of short-range FM correlations close to the transition temperature. Below the magnetic transition, the $M(H)$ data continue to show nearly linear behavior, as expected for an antiferromagnet. Although the magnetic state of the sample is mainly antiferromagnetic, the deviation from linearity at low fields indicates that some ferromagnetic order is also present in the system. It is worth mentioning that the $M(T)$ and $M(H)$ behavior measured in the [110] and [1 $1 \overline{0} 0]$ directions behaves similar to that reported for the [001] direction.

The detailed view (low fields) of the magnetization versus field curves at the selected temperatures is shown in Fig. 3, along the three crystallographic directions (a) [001], (b) [110], and (c) [1ํㅣㄹ. The magnetization data at $350 \mathrm{~K}$ show

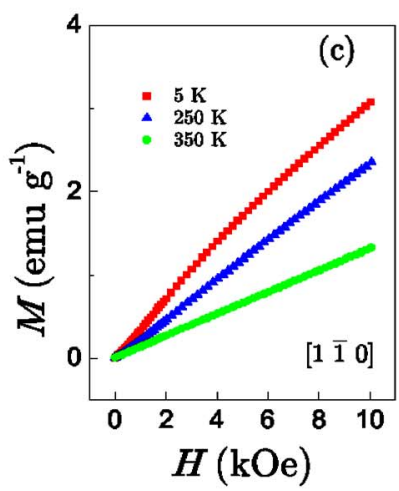

FIG. 3. (Color online) Magnetization versus field curves for small fields along three crystallographic directions (a) [001], (b) [110], and (c) [1 $\overline{10}]$ at selected temperatures. The dashed lines in (a) represent the linear behavior extrapolated at small and high fields at $5 \mathrm{~K}$. The inset shows the deviation of the magnetization from the linear behavior corresponding to the small-field region.

the typical linear behavior for a paramagnetic state. By contrast, below the magnetic transition, the $M(H)$ curves deviate from linearity at small fields but recover the linear behavior at fields higher than $5 \mathrm{kOe}$. Such a behavior is illustrated in Fig. 3(a) where the dashed lines represent the linear behavior extrapolated at small and high fields at $5 \mathrm{~K}$. The inset shows the deviation of the magnetization from linear behavior. It is seen that the deviation saturates at about $5 \mathrm{kOe}$. This behavior indicates the presence of some degree of ferromagnetic order, which is in agreement with previous magnetic characterization of Ni-Mn-Al. ${ }^{21-23}$

By comparing the temperature dependence of the elastic constants [Figs. 1(a) and 1(b)] and magnetization [Fig. 2(a)] it is seen that the deviation from Debye behavior occurs within the temperature range of the magnetic transition. In the vicinity of the magnetic transition, the elastic constants are influenced by the onset of magnetic order showing a reduction of $C_{L}$ and $C_{44}$. The magnetic contribution to the elastic constants, obtained as the difference between the normal Debye behavior extrapolated from the paramagnetic region and the measured value, is negative for both modes. This behavior is in contrast with that displayed by the Ni$\mathrm{Mn}-\mathrm{Ga}$ ferromagnetic system which, in the absence of magnetic field, shows no significant change in any elastic moduli at the Curie point. ${ }^{13}$ We note that deviations from Debye behavior have been observed in antiferromagnetic systems such as chromium ${ }^{30}$ and Mn-Invar alloys ${ }^{31,32}$ below their Néel temperatures.

\section{Magnetic-field dependence of the elastic constants}

To investigate further the interplay between elastic and magnetic properties, we have measured the elastic constants
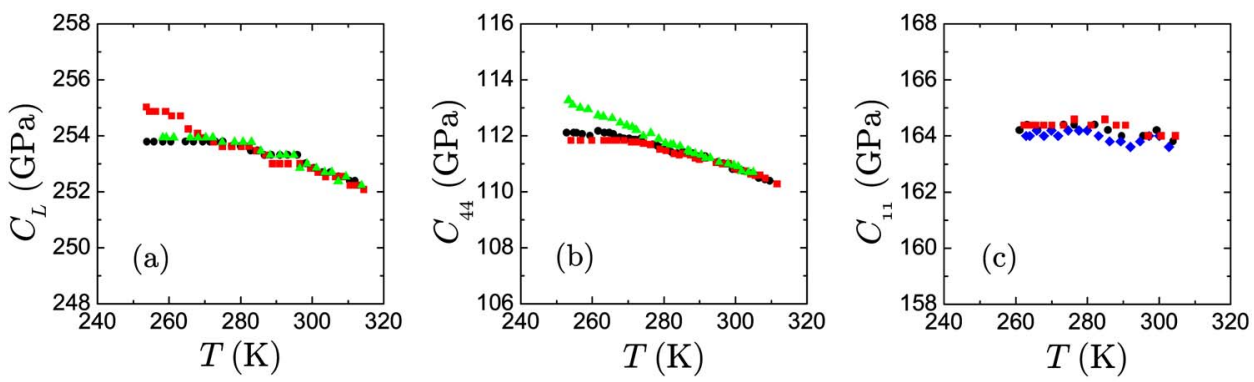

FIG. 4. (Color online) Temperature dependence of the elastic constants measured while cooling across the magnetic transition under magnetic field $H=5 \mathrm{kOe}$ (a) $C_{L}$, (b) $C_{44}$, and (c) $C_{11}$. Circles represent the values without applied magnetic field. Square, triangle, and diamond symbols represent measurements obtained with the magnetic field applied along the [1 10$]$, [001], and [110] directions, respectively. 

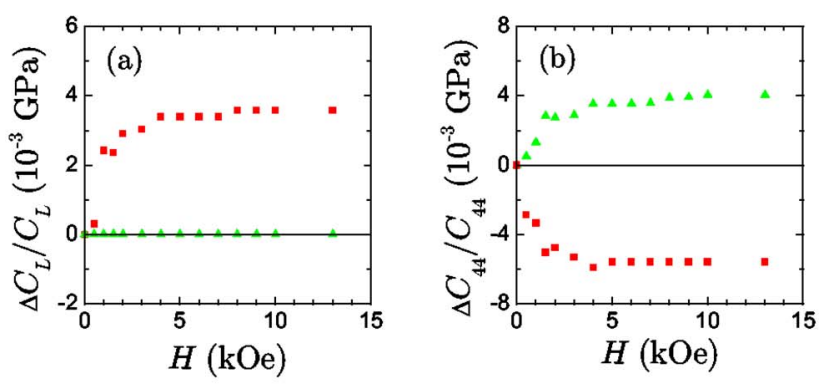

under isofield and isothermal conditions. Application of a magnetic field caused strong distortion of the ultrasonic echoes of the [110] [1 $1 \overline{1} 0]$ mode, and, consequently, it was not possible to measure the magnetic-field dependence of $C^{\prime}$. Figure 4 shows the temperature dependence of the elastic constants measured under an applied magnetic field of $H$ $=5 \mathrm{kOe}$ along different crystallographic directions perpendicular to the propagation direction of the ultrasonic waves. The data correspond to cooling runs through the magnetic transition, both without a magnetic field and with an applied field. Figures 4(a) and 4(b) show, respectively, the values of $C_{L}$ and $C_{44}$ measured along the [110] propagation direction (circles). Square symbols represent measurements obtained with the magnetic field applied along the [1 $1 \overline{1} 0]$ direction, and triangle symbols represent those obtained with the magnetic field along the [001] direction. Figure 4(c) shows the values of $C_{11}$ measured along the [001] propagation direction (circles). The diamond symbols correspond to measurements obtained with the field along the [110] direction. From the figure, it is seen that the three elastic constants behave differently when a magnetic field is applied. While $C_{11}$ is not affected by the magnetic field, $C_{L}$ and $C_{44}$ exhibit different behavior with and without applied field. On the one hand, the value of $C_{L}$ increases for a field along the [1 $\left.\overline{1} 0\right]$ direction, while it is not affected by a field along the [001] direction. On the other hand, $C_{44}$ decreases for a field along the [1피 direction and increases for a field along the [001] direction. The different behavior exhibited by the elastic constants evidences an anisotropic magnetoelastic coupling for Ni-Mn-Al.

The behavior found for the temperature dependence of the elastic constants under magnetic field is confirmed by complementary isothermal measurements. The relative change of the elastic constants with respect to the value at zero field at $T=263 \mathrm{~K}$ is presented in Fig. 5. $C_{11}$ is not affected by a magnetic field, while $C_{L}$ and $C_{44}$ change when a field is applied. This is consistent with the measurements performed on cooling through the magnetic transition temperature with applied magnetic field; $C_{L}$ increases up to saturation for a field along the [1 $1 \overline{1} 0]$ direction, while it is not affected by a field along the [001] direction; $C_{44}$ decreases down to saturation for a field along the $[1 \overline{1} 0]$ direction and increases up to a saturation for a field along the [001] direc-

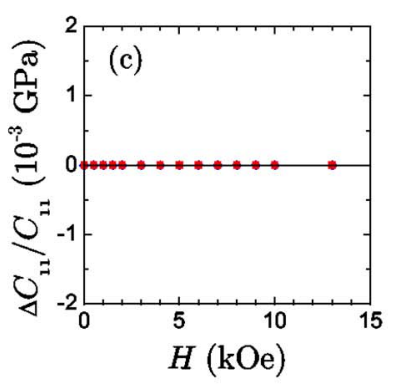

FIG. 5. (Color online) Magnetic-field dependence of the relative change of the elastic constants with respect to the value at zero field at $T=263 \mathrm{~K}$. Square, triangle, and diamond symbols represent measurements obtained with the magnetic field applied along the [1힐 $[001]$, and [110] directions, respectively.

tion. In the ferromagnetic Ni-Mn-Ga system, all elastic constants increase up to a saturation value with increasing magnetic field. ${ }^{15}$ Note that all relative changes saturate at a field of approximately $5 \mathrm{kOe}$. Comparison with the behavior of the magnetization at small fields (Fig. 3) shows that the behavior of the relative change of the elastic constants is related to nonlinearities observed in low fields in the magnetization versus field curves. These also saturate at $5 \mathrm{kOe}$.

\section{SUMMARY AND CONCLUSIONS}

Ultrasonic methods have been used to determine the elastic constants of a Ni-Mn-Al Heusler alloy over a broad temperature range. The room-temperature values are in excellent agreement with the values obtained from the slopes of the phonon dispersion curves. The low-temperature bulk modulus agrees well with the reported value for antiferromagnetic $\mathrm{Ni}_{2} \mathrm{MnAl}$ from $a b$ initio calculations. This gives experimental confirmation for the softening to volume changes in the antiferromagnetic state with respect to the harder ferromagnetic state. A deviation from Debye behavior has been observed below the magnetic transition for all modes. Such a softening of the lattice is a result of magnetoelastic coupling. Elastic constant measurements under magnetic field indicate the existence of an anisotropic magnetoelastic coupling. Such a coupling saturates at about $5 \mathrm{kOe}$ for all modes and is associated with the nonlinear behavior at low fields exhibited by the magnetic-field dependence of the magnetization.

\section{ACKNOWLEDGMENTS}

We acknowledge Sophie Guézo for her collaboration. This work received financial support from the CICyT (Spain), Project No. MAT2004-01291, Marie-Curie RTN MULTIMAT (EU), Contract No. MRTN-CT-2004-505226, DURSI (Catalonia), Project No. 2005SGR00969, and from the Deutsche Forschungsgemeinschaft (GK277). X.M. acknowledges support from DGICyT (Spain). Ames Laboratory is operated for the U.S. Department of Energy by Iowa State University under Contract No. W-7405-Eng-82. The work at Ames was supported by the Director for Energy Research, Office of Basic Energy Sciences. 
*Electronic address: 1luis@ecm.ub.es

${ }^{1}$ Magnetism and Structure in Functional Materials, edited by A. Planes, L. Mañosa, and A. Saxena, Materials Science Series, Vol. 79 (Springer-Verlag, Berlin, 2005).

${ }^{2}$ R. C. O’Handley, J. Appl. Phys. 83, 3263 (1998).

${ }^{3}$ F. Hu, B. Shen, and J. Sun, Appl. Phys. Lett. 76, 3460 (2000).

${ }^{4}$ J. Marcos, A. Planes, L. Mañosa, F. Casanova, X. Batlle, A. Labarta, and B. Martínez, Phys. Rev. B 66, 224413 (2002).

${ }^{5}$ T. Krenke, E. Düman, M. Acet, E. F. Wasserman, X. Moya, L. Mañosa, and A. Planes, Nat. Mater. 4, 450 (2005).

${ }^{6}$ K. Ullakko, J. K. Huang, C. Kanter, R. C. O’Handley, and V. V. Kokorin, Appl. Phys. Lett. 69, 1966 (1996).

${ }^{7}$ A. Sozinov, A. A. Likhachev, N. Lanska, and K. Ullakko, Appl. Phys. Lett. 80, 1746 (2002).

${ }^{8}$ A. Zheludev, S. M. Shapiro, P. Wochner, A. Schwartz, M. Wall, and L. E. Tanner, Phys. Rev. B 51, 11310 (1995).

${ }^{9}$ U. Stuhr, P. Vorderwisch, V. V. Kokorin, and P.-A. Lindgård, Phys. Rev. B 56, 14360 (1997).

${ }^{10}$ L. Mañosa, A. Planes, J. Zarestky, T. Lograsso, D. L. Schlagel, and C. Stassis, Phys. Rev. B 64, 024305 (2001).

${ }^{11}$ J. Worgull, E. Petti, and J. Trivisonno, Phys. Rev. B 54, 15695 (1996).

${ }^{12}$ L. Mañosa, A. Gonzàlez-Comas, E. Obradó, A. Planes, V. A. Chernenko, V. V. Kokorin, and E. Cesari, Phys. Rev. B 55, 11068 (1997).

${ }^{13}$ M. Stipcich, L. Mañosa, A. Planes, M. Morin, J. Zarestky, T. Lograsso, and C. Stassis, Phys. Rev. B 70, 054115 (2004).

${ }^{14}$ A. Planes, E. Obradó, A. Gonzàlez-Comas, and L. Mañosa, Phys. Rev. Lett. 79, 3926 (1997).

${ }^{15}$ A. Gonzàlez-Comas, E. Obradó, L. Mañosa, A. Planes, V. A. Chernenko, B. J. Hattink, and A. Labarta, Phys. Rev. B 60, 7085 (1999).
${ }^{16}$ S. Morito, T. Kakeshita, K. Hirata, and K. Otsuka, Adv. Mater. (Weinheim, Ger.) 46, 5377 (1998).

${ }^{17}$ S. Morito and K. Otsuka, Mater. Sci. Eng., A 208, 47 (1996).

${ }^{18}$ J. Pons, V. A. Chernenko, R. Santamarta, and E. Cesari, Adv. Mater. (Weinheim, Ger.) 48, 3027 (2000).

${ }^{19}$ T. Krenke, M. Acet, E. F. Wassermann, X. Moya, L. Mañosa, and A. Planes, Phys. Rev. B 72, 014412 (2005).

${ }^{20}$ T. Krenke, M. Acet, E. F. Wassermann, X. Moya, L. Mañosa, and A. Planes, Phys. Rev. B 73, 174413 (2006).

${ }^{21}$ M. Acet, E. Duman, E. F. Wassermann, L. Mañosa, and A. Planes, J. Appl. Phys. 92, 3867 (2002).

${ }^{22}$ L. Mañosa, A. Planes, M. Acet, E. Duman, and E. F. Wassermann, J. Appl. Phys. 93, 8498 (2003).

${ }^{23}$ J. Y. Rhee, Y. V. Kudryavtsev, and Y. P. Lee, J. Magn. Magn. Mater. 272-276, e245 (2004).

${ }^{24}$ K. R. A. Ziebeck and P. J. Webster, J. Phys. F: Met. Phys. 5, 1756 (1975).

${ }^{25}$ X. Moya, L. Mañosa, A. Planes, T. Krenke, M. Acet, O. V. Garlea, T. A. Lograsso, D. L. Schlagel, and J. Zarestky, Phys. Rev. B 73, 064303 (2006).

${ }^{26}$ S. C. Lakkad, J. Appl. Phys. 42, 4277 (1971).

${ }^{27}$ A. Planes and L. Mañosa, Solid State Phys. 55, 159 (2001).

${ }^{28}$ A. Planes, L. Mañosa, and E. Vives, Phys. Rev. B 53, 3039 (1996).

${ }^{29}$ T. Büsgen, J. Feydt, R. Hassdorf, S. Thienhaus, M. Moske, M. Boese, A. Zayak, and P. Entel, Phys. Rev. B 70, 014111 (2004).

${ }^{30}$ W. C. Muir, J. M. Perz, and E. Fawcett, J. Phys. F: Met. Phys. 17, 2431 (1987).

${ }^{31}$ M. Cankurtaran, G. A. Saunders, P. Ray, Q. Wang, U. Kawald, J. Pelzl, and H. Bach, Phys. Rev. B 47, 3161 (1993).

${ }^{32}$ U. Kawald, O. Mitze, H. Bach, J. Pelzl, and G. A. Saunders, J. Phys.: Condens. Matter 6, 9697 (1994). 